\title{
Design of Buck-Boost Semiconductor Lighting Drive Based on Multi-loop Control
}

\author{
Renbo $\mathrm{Xu}^{1.2} \mathrm{Jie} \mathrm{Li}^{1}$ and Huanhuan Chen ${ }^{1}$ \\ ${ }^{1}$ School of Electrical and Information Engineering, Nanchang Institute of Science \& Technology, \\ ${ }^{2}$ School of Physics and Microelectronics, Central South University
}

Keywords: Semiconductor lighting; Buck-Boost, OCC multi-loop control; Efficient drive

\begin{abstract}
Semiconductor lighting has the characteristics of energy saving, high efficiency, long life, environmental protection and so on. It is the product of "green lighting" concept and semiconductor light-emitting technology. Based on the condition that OCC (One Cycle Control) controls Buck-Boost type semiconductor lighting drive, a multi-loop control strategy is proposed, and a new type of efficient lighting drive is obtained. The multi-loop control strategy is deeply analyzed, such as Simulink, SimPowerSystems tool and the main circuit small-signal model modeling and simulation. From the system simulation, it can be seen that multi-ring control strategy has a higher power factor, lower output impedance and better dynamic performance, comparing with the OCC control strategy.
\end{abstract}

\section{Introduction}

Semiconductor lighting has the characteristics of energy saving, high efficiency, long life, environmental protection and others, and it's another lighting source revolution[1-4] after incandescent, fluorescent lamp and high pressure gas discharge lamp. As shown in Fig. 1, it's a commonly used Buck-Boost type semiconductor lighting drive controlled by OCC, and the response speed, tracking and control ability, anti-input disturbance ability is strong [5-6], but because of the dynamic characteristics of the filter, the output voltage waveform of the whole drive system is disturbed and the running performance [7-8] of the drive system is reduced.

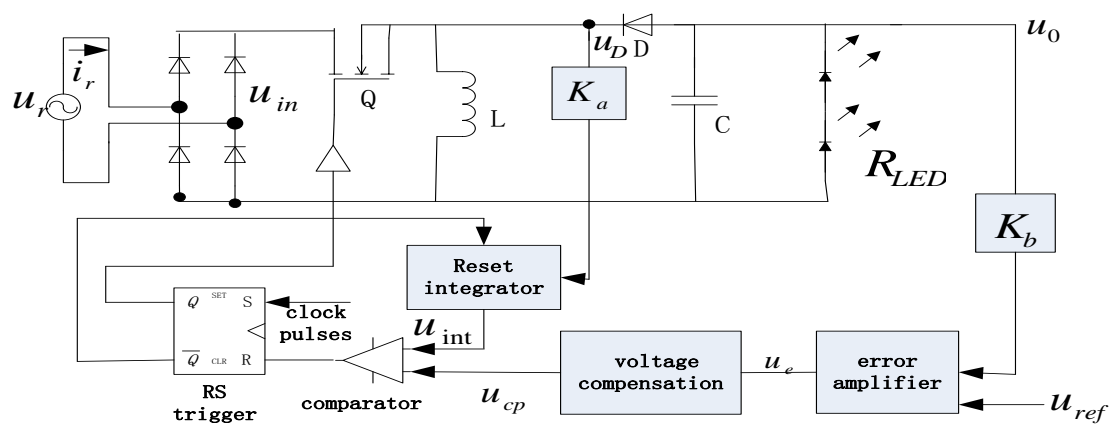

Figure 1. OCC controlling Buck-Boost type semiconductor lighting drive

\section{Working Principle Analysis of Multiple Loop Control Drive}

In order to make the Buck-Boost drive get higher power factor, improve the anti-input interference and anti-load disturbance ability, a new multi-loop control method is proposed based on the OCC control strategy to obtain the multi-loop control Buck-Boost type drive, as shown in Fig. 2. Compared with the OCC controlled Buck-Boost LED lighting drive, a loop composed of voltage 
differential, gain $K_{c}$ and adder is added from the structure, plus $K_{a}, K_{b}$ two loop controlled by OCC, , that is, multi-loop control.

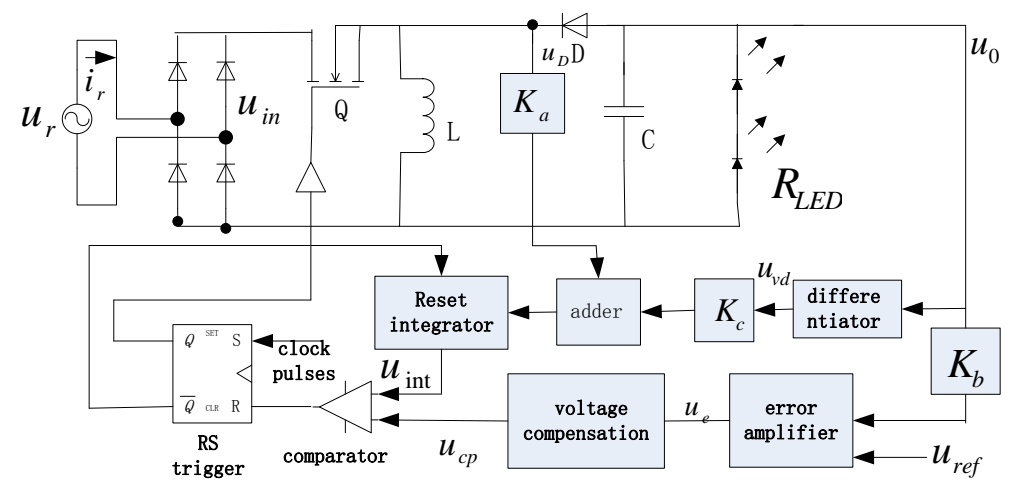

Figure 2. Multi-loop control Buck-Boost drive circuit

In fig. 2, the $\mathrm{AC}$ input voltage is ${ }_{r}$, the $\mathrm{AC}$ input current is ${ }^{i}$. The input voltage passes the diode rectifier to get voltage ${ }^{u_{i n}}$, and the switch tube $Q$ is controlled by the RS flip-flop, and when the pulse rising time comes, the same phase of RS flip-flop will give the switch a high level, and the switch is turned on and the output voltage $u_{o}$ is sampled to obtain a voltage signal $K_{b} u_{o}$, which is sent to the error amplifier together with the reference voltage signal ${ }^{u_{\text {ref }}}$. The resulting error signal $u_{e}$ gets the signal $u_{c p}$ and sends it to the opposite side of the comparator after voltage compensation. The voltage signal $u_{D}$ gets the signal $K_{a} u_{D}$ after sampling and transmits it to the adder. The other input of the adder is the voltage signal $K_{c} u_{v d}$ after the differentiator and gain $K_{c} u_{v d}$. The adder output is connected to the integrator with reset switch, and the integrator output $u_{\text {int }}$ outputs to the comparator same-phase. When the integrated voltage signal $u_{i n t}$ rises to the compensated voltage signal $u_{c p}$, the comparator reverses and output high level, the RS flip-flop is reset, the same-phase terminal outputs low level, the switch is turned off, the inverting terminal outputs a high level, making reset integrator reset until the next clock pulse arrives, the switch is turned on again.

\section{Establishment of System Model and Simulation Analysis}

System Model. Combined with the Buck-Boost drive main circuit and multi-loop control principle analysis, the system model of multi-loop control Buck-Boost drive can be got, as shown in the following fig. 3 :

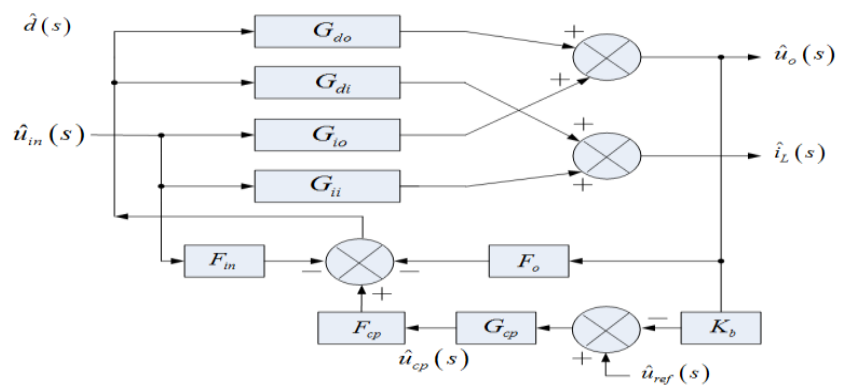

Figure 3. Multi-loop control Buck-Boost type driving system 
From the multi-loop control Buck-Boost type driven system model fig. 3 multi-loop, Mason formula[9-10] can be simplified to get input and output transfer function:

$$
G_{\text {uio }}=\frac{\hat{u}_{o}(s)}{\hat{u}_{i n}(s)}=\frac{G_{i o}-F_{i n} G_{d o}}{1-F_{o} G_{d o}}=0
$$

It can be seen that even if the input voltage has a large disturbance, the output voltage will not be affected, that is, multi-loop control Buck-Boost drive system has a good anti-input interference. Further analysis can get control output transfer function and the system loop gain function of multi-loop control convertor, as follows:

$$
\begin{aligned}
& G_{\text {uco }}=\frac{\hat{u}_{o}(s)}{\hat{u}_{c p}(s)}=\frac{\left(1+R_{b} C_{b} s\right)\left[L D s-R(1-D)^{2}\right]}{K_{a}\left(1+R_{b} C_{b} s\right)\left[R L C(1-D)^{2} s^{2}+L(1-D)^{2} s+R(1-D)^{4}\right]+K_{c} D R_{b} C_{b} s\left[L D s-R(1-D)^{2}\right]} \\
& G_{\text {loop }}=K_{b} G_{c p} G_{\text {uco }}=\frac{K_{b} G_{c p}\left(1+R_{b} C_{b} s\right)\left[L D s-R(1-D)^{2}\right]}{K_{a}\left(1+R_{b} C_{b} s\right)\left[R L C(1-D)^{2} s^{2}+L(1-D)^{2} s+R(1-D)^{4}\right]+K_{c} D R_{b} C_{b} s\left[L D s-R(1-D)^{2}\right]}
\end{aligned}
$$

Simulation Proof. In order to verify the validity and rationality of multi-loop control strategy Buck-Boost driver proposed in this paper. Simulink and SimPowerSystems tools in MATLAB are used to set up the main circuit model and multi-loop control model of Buck-Boost drive converter respectively, connected in the form of the same name of Input and output ports, as shown in Fig. 4, Fig. 5. The main parameters of the circuit are:

Input voltage: $90 \sim 270 \mathrm{~V}$ (Rated: $220 \mathrm{~V}$ )

Switch frequency: $100 \mathrm{kHz}$

Output voltage: $40 \mathrm{~V}$

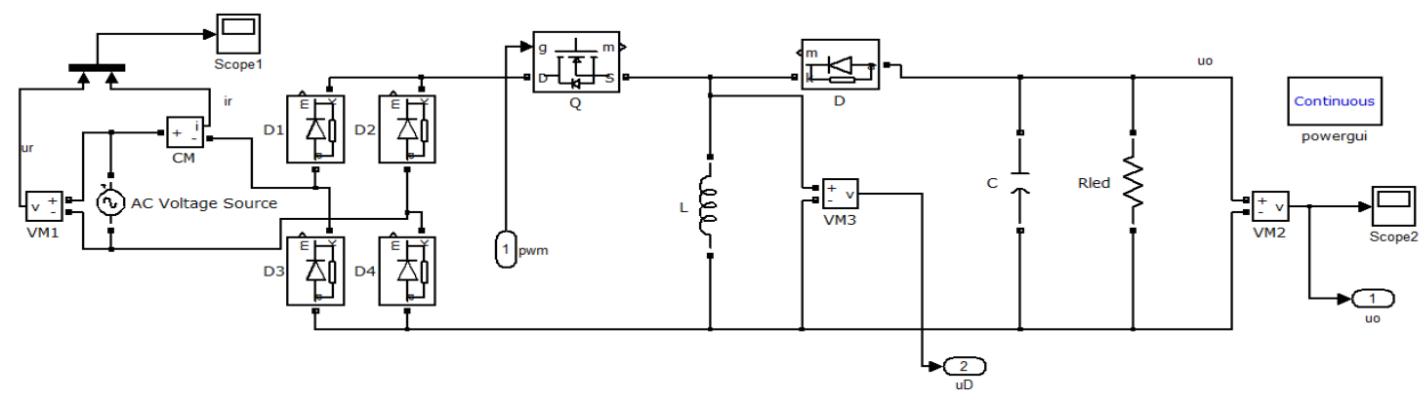

Figure 4. Main circuit model of Buck-Boost driver

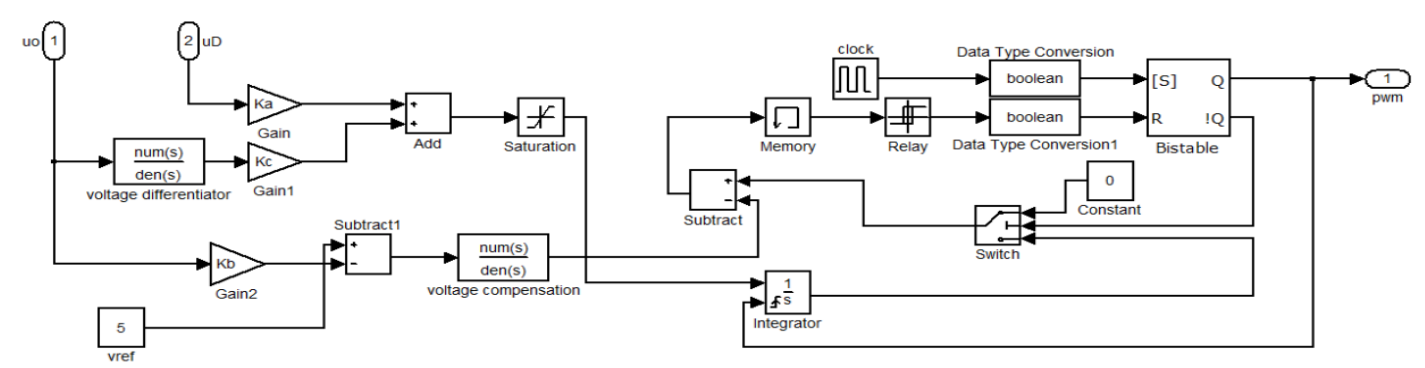

Figure 5. Multi-loop control model 
As shown in Fig. 6, we can see that the input voltage is $220 \mathrm{~V}, 50 \mathrm{~Hz}$ sine wave, the input current waveform is approximately sinusoidal, that is, the input current well tracks the input voltage, the total harmonic distortion is small, and the power factor is high. Fig. 7 shows the oscilloscope Scope 2 system output voltage waveform, visible system responding fast, overshoot smaller. The output voltage is stable to $-40 \mathrm{~V}$, to achieve reverse polarity output, in line with the input and output voltage relationship.

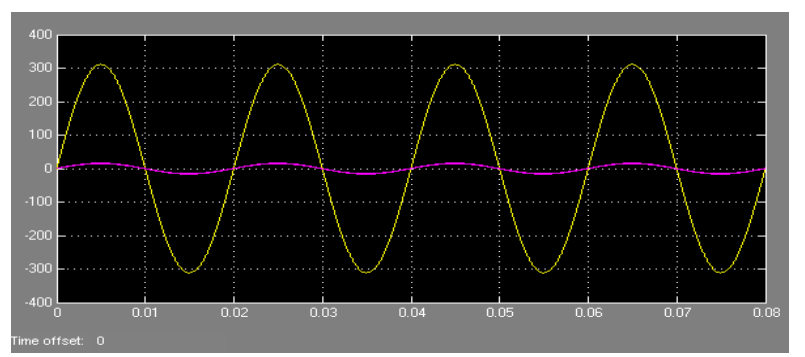

Figure 6. AC input voltage, input current waveform

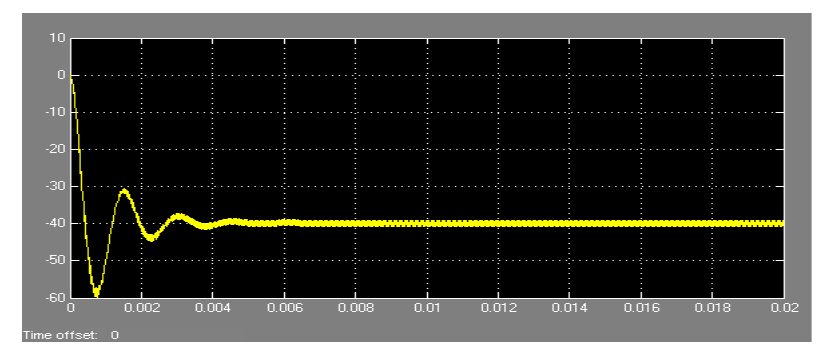

Figure 7. System output voltage waveform

In order to verify the anti-input voltage disturbance ability of multi-loop control converter, a square wave voltage signal disturbance with amplitude of $25 \mathrm{~V}$ and period of $0.004 \mathrm{~s}$ is applied at $0.01 \mathrm{~s}$, as shown in Fig. 8, when the input voltage is disturbed, and the output voltage is not affected and continues to remain stable output.

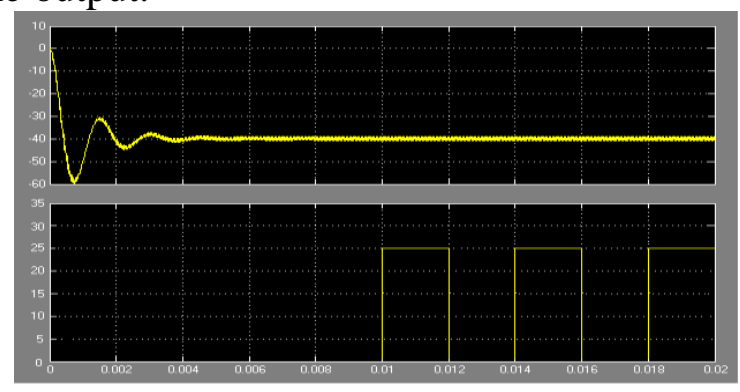

Figure 8. Analysis of Input Voltage Disturbance

In order to verify the anti-load disturbance ability of multi-loop control converter, the load current instantly jumps from $1 \mathrm{~A}$ to $0.8 \mathrm{~A}$ at $0.01 \mathrm{~s}$. As shown in Fig. 9, when the load current jumps, the output voltage waveform also produces a larger jump. But after a relatively short period, the output voltage restores stability, that is, multi-loop control Buck-Boost drive converter has a good dynamic load performance. 


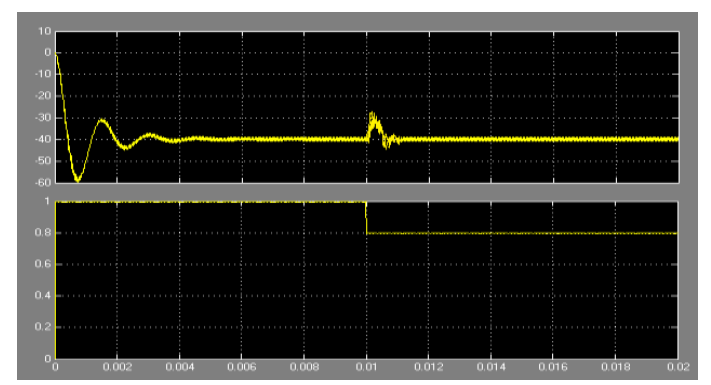

Figure 9. Analysis of Anti-load Disturbance

\section{Conclusion}

Based on OCC controlling Buck-Boost driver, this paper proposes a new multi-loop control strategy, which is analyzed and deduced and carried out modeling and simulation through Simulink and SimPowerSystems. Compared with the OCC control strategy, multi-loop control Buck -Boost-type drive has smaller total harmonic distortion, higher power factor, faster system response speed, smaller overshoot, stable output voltage, and high resistance to input voltage and anti-load disturbance.

\section{Acknowledgements}

Funding project: Science Research Key Project of Jiangxi Provincial Education Department (Project No .: GJJ161218).

\section{References}

[1] Y. Hu, M M, Jovanovic. LED Driver with Self-Adaptive Drive Voltage. IEEE Transactions on Power Electronics, 2008, 23(6): 116 125

[2] Y.K. Cheng, K.W.E. Cheng, General Study for Using LED to replace Traditional Lighting Devices. 2nd International Conference on Power Electronics Systems and Applications, Hong Kong, 2012, 25(9):173 177

[3] H. van der Broeck, G. Sauerlander, M. Wendt. Power Driver Topologies and Control Schemes for LEDs. IEEE Applied Power Electronics Conference, Anaheim, 2007， 31(5):319 325

[4] B Wang, X Ruan and K Yao. A Method of Reducing the Peak- to -Average Ratio of LED Current for Electrolytic Capacitor-Less AC-DC Drivers. IEEE Transactions on Power Electronics, 2010, 25(3): 592 601

[5] M Dyble, Narendran $\mathrm{N}$ and Bierman A. Impact of dimming white LEDs: chromaticity shifes due to different dimming methods. Proc. SPIE, 2008, 35(4):1 9

[6] K.H Wu Chou S, A simple current-balancing converter for LED lighting. IEEE Applied Power Electronics Conference, Washington DC, USA, 2009， 35(9):16 19

[7] M. Nishikawa, K. Morihori and Y. Ishizuka. Static characteristics of a drive circuit for LED with constant-current control. Annual Conference of IEI-J, 2009，32(7):251 253

[8] L Gu, X Ruan, M Xu and K Yao, Means of Eliminating Electrolytic Capacitor in AC/DC Power Supplies for LED Lightings. IEEE Trans. Power Electronics, 2009, 24(5): 399 408

[9] H.J Chiu, Y.K Lo and J.T Chen, A high-Efficiency Dimmable LED Driver for Low-Power Lighting Applications. IEEE Transactions on Industrial Electronics, 2010, 57(2): 735 743

[10] Y.Q Hu, Jovanovic and Milan M. A Novel LED Driver with Adaptive Drive Voltage. Applied Power Electronics Conference and Exposition, 2011, 37(6):565 571. 